\title{
Detection of K-ras gene mutations in feces by magnetic nanoprobe in patients with pancreatic cancer: A preliminary study
}

\author{
XIAOGUANG WANG $^{1^{*}}$, JINGSHUAI WANG ${ }^{2 *}$, FEI CHEN $^{1}$, ZHENGXIANG ZHONG $^{1}$ and LIFENG QI ${ }^{2,3}$ \\ ${ }^{1}$ Department of Surgery, Jiaxing Second Hospital, Jiaxing, Zhejiang 314000; ${ }^{2}$ Institute for Biomedical Engineering and \\ Nano Science, Tongji University School of Medicine, Shanghai 200092; ${ }^{3}$ Foxgene Co. Ltd., Wuxi, Jiangsu 200014, P.R. China
}

Received May 11, 2016; Accepted March 24, 2017

DOI: $10.3892 /$ etm.2017.5368

\begin{abstract}
The present study aimed to investigate the feasibility and effectiveness of detecting K-ras mutation by using magnetic nanoparticles in fecal samples of patients with pancreatic cancer at different stages. The novel methodology of K-ras mutation detection was compared to the existing methodology of cancer antigen (CA)19-9 examination. Patients with pancreatic cancer $(n=88)$, pancreatic benign diseases who displayed chronic pancreatitis $(n=35)$, pancreatic mucinous cyst neoplasms $(n=10)$ and pancreatic serous cyst $(n=9)$ admitted to the Department of Surgery, Jiaxing Second Hospital were enrolled in the present study. Fecal samples were collected from all patients, DNA was extracted and magnetic nanoprobe was then used to detect K-ras mutation. The results obtained using the novel magnetic nanoprobe detection technique showed a K-ras mutation rate of $81.8 \%(72 / 88)$ in the patients with pancreatic cancer and $18.5 \%(10 / 54)$ in patients with pancreatic benign diseases. In patients with pancreatic cancer, the K-ras mutation rate was comparable in stages I + IIA and IIB + III + IV (78.9 vs. $84.0 \%$; P>0.05). The sensitivity and specificity of K-ras mutation for detection of pancreatic cancer was 81.8 and $81.5 \%$, respectively. Sixty-eight pancreatic cancer patients had $>37 \mathrm{U} / \mathrm{ml}$ CA99 with a sensitivity and specificity for pancreatic cancer detection of 77.3 and $77.8 \%$, which was not significantly lower than detection by the fecal K-ras mutations ( $P>0.05)$. Combinational detection of fecal K-ras mutations and serum CA19-9 significantly increased the sensitivity
\end{abstract}

Correspondence to: Dr Zhengxiang Zhong, Department of Surgery, Jiaxing Second Hospital, 1518 Huanchengbei Road, Jiaxing, Zhejiang 314000, P.R. China

E-mail: zhongzhengxiang1@sina.com

Professor Lifeng Qi, Institute for Biomedical Engineering and Nano Science, Tongji University School of Medicine, 1239 Siping Road, Shanghai 200092, P.R. China

E-mail: 1fqi@tongji.edu.cn

*Contributed equally

Key words: pancreatic cancer, K-ras, point mutation, fecal DNA, magnetic nanoparticles, magnetic nanoprobe regarding pancreatic cancer detection to $97.7 \%(\mathrm{P}<0.05)$, while the specificity was not enhanced $(80.9 \% ; \mathrm{P}>0.05)$ compared with fecal K-ras mutations or CA19-9 alone. The findings showed that the magnetic nanoprobe is able to detect fecal $\mathrm{K}$-ras mutations in different stages of pancreatic cancer, with comparable sensitivity and specificity to CA19-9 examination for differentiating pancreatic cancer. Furthermore, combined detection of CA19-9 and K-ras mutations has enhanced sensitivity compared with CA19-9 alone.

\section{Introduction}

Pancreatic cancer is one of the most malignant types of gastrointestinal tract tumor with a 5-year survival rate of $<5 \%$ (1). In recent years, the incidence of pancreatic cancer has been increasing. Early detection of pancreatic cancer is difficult due to its nonspecific clinical manifestations and therefore, a large proportion of patients are diagnosed at the advanced stage beyond the time window for radical surgery (2). Cancer antigen (CA)19-9 is the main tumor biomarker for diagnosing pancreatic cancer with a reported sensitivity of $65 \%$ and a specificity of 78-94\% $(3,4)$. Considering that CA19-9 cannot be used as an early detection marker for pancreatic cancer and is also detected in benign pancreatobiliary diseases, particularly chronic pancreatitis (5), its specificity and effectiveness may not be satisfactory. Thus, there is a requirement for developing novel methodologies to detect pancreatic cancer at different stages and make earlier diagnoses, which will ultimately lead to a better prognosis for patients.

With the development of modern molecular biological techniques, numerous novel and efficient detection methods have been developed, including those for the detection of tumor-associated genetic mutations, which is also a current focus of research on pancreatic cancer. The K-ras gene is closely associated with the development and progression of pancreatic cancer. K-ras mutation is an important and early event in tumorigenesis (6-8). K-ras is able to bind guanine nucleotides within a growth factor signal transduction pathway, while pathological mutations of K-ras lead to cell proliferation (9). The detection of K-ras mutations in biopsy specimens, pancreatic juice, bile and blood has been previously reported $(10,11)$. Genetic mutations detected in biopsy specimens and pancreatic juice may be a reliable method for diagnosing pancreatic cancer; however, it is 
Table I. Fecal K-ras mutations in pancreatic cancer, pancreatic benign diseases and healthy control group.

\begin{tabular}{lccrr}
\hline Group & Cases $(\mathrm{n})$ & Cases carrying K-ras mutations, $\mathrm{n}(\%)$ & $\chi^{2}$ & P-value \\
\hline Pancreatic cancer & 88 & $72(81.8)$ & & \\
Pancreatic benign diseases & 54 & $10(18.5)$ & 54.954 & $<0.001^{\mathrm{a}}$ \\
Healthy control & 31 & $0(0)$ & 5.043 & $0.025^{\mathrm{a}}$ \\
\hline
\end{tabular}

${ }^{\mathrm{a}} \mathrm{P}<0.05$ vs. pancreatic cancer group.

challenging to obtain adequate samples (12). Conversely, K-ras detection in blood has a relatively low sensitivity of $66-71 \%$ and requires combination with other examinations to increase the diagnostic rate (13-15). Recent studies have demonstrated that it is possible to extract and sequence fecal DNA $(16,17)$. However, K-ras mutation has not been investigated in pancreatic cancer by magnetic nanoprobe. The present study introduced a nanoparticle trace capture probe, which is widely applied in detecting trace genetic variants $(18,19)$, to detect K-ras mutations in the feces of patients with pancreatic cancer at different stages and further explored the sensitivity and specificity of the novel K-ras mutation detection method and the existing CA19-9 examination method as well as their combination regarding pancreatic cancer diagnosis.

\section{Patients and methods}

Patients. Patients with pancreatic diseases admitted to the Department of Surgery, Jiaxing Second Hospital (Jiaxing, China) from January 2013 to August 2015 were enrolled in the present study, including patients with diagnoses of pancreatic cancer $(n=88)$, chronic pancreatitis $(n=35)$, pancreatic mucinous cyst $(n=10)$ and pancreatic serous cyst $(n=9)$. Thirty one healthy individuals were also included as controls. Pancreatic tissue was obtained from patients after surgical resection at the Department of Surgery, Jiaxing Second Hospital. Tissue samples were obtained at the time of resection, during analysis of frozen sections or both, in accordance with the in-house protocol. Clinicopathological [i.e., clinical manifestation, tumor location, CA19-9, carcino-embryonic antigen (CEA)] and demographic data (i.e., age, sex, histology and tumor stage) were collected and analyzed. Informed consent was obtained from all the patients and the present study was approved by the Ethics Committee of Jiaxing Second Hospital.

DNA extraction from fecal specimens. Fecal samples were stored at $-80^{\circ} \mathrm{C}$. DNA was extracted by phenol-chloroform extraction from $200 \mathrm{mg}$ feces and purified using a Qiagen purification kit (Qiagen, Hilden, Germany). DNA concentrations were measured using a ND-1000 NanoDrop (Thermo Fisher Scientific, Inc., Waltham, MA, USA).

Establishment of nanoparticle trace capture probe system. A solution of $1 \mathrm{M} \mathrm{NaOH}$ (Sigma-Aldrich; Merck KGaA, Darmstadt, Germany) and 0.1 M salicylic acid (SA, Sigma-Aldrich; Merck KGaA) with volume at $100 \mu 1$ each was added to a sterilized three-necked flask to increase the $\mathrm{pH}$ of the

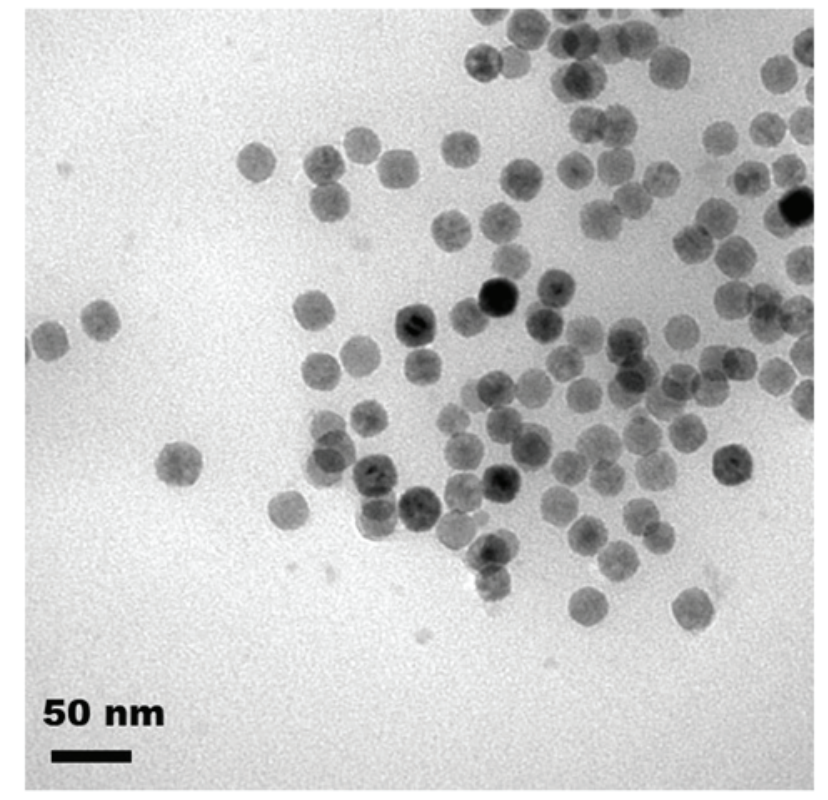

Figure 1. Morphology of magnetic nanoparticles by electron microscopy.

whole reaction solution to $~ 11.0$ under vigorous stirring in an argon gas atmosphere, followed by the addition of an aqueous solution comprising a mixture of $\mathrm{Fe}(\mathrm{III})$ and $\mathrm{Fe}(\mathrm{II})$ oxide salts $\left(\mathrm{Fe}_{2} \mathrm{O}_{3}\right.$ and $\mathrm{Fe}_{3} \mathrm{O}_{4}$; Merck $\left.\mathrm{KGaA}\right)$ with a molar ratio of $2 \mathrm{X}$ $\mathrm{Fe}(\mathrm{III}) / 1 \mathrm{X} \mathrm{Fe}(\mathrm{II}) / 4 \mathrm{X}$ SA until a black suspension was obtained. After refluxing at $90^{\circ} \mathrm{C}$ for $4 \mathrm{~h}$, a dark-brown suspension was formed. The morphology of the magnetic nanoparticles was observed by transmission electron microscopy (Fig. 1).

Detection of K-ras using magnetic nanoparticles. A capture probe for K-ras (10 $\mu \mathrm{M}$, Foxgene Co., Ltd., Wuxi, China) was conjugated with $1 \mu \mathrm{M}$ magnetic nanoparticles by 1-ethyl-3-(-3-dimethylaminopropyl) carbodiimide hydrochloride chemistry (Sigma-Aldrich; Merck KGaA) and purified by a magnetic field, followed by redispersion in Tris-EDTA buffer (20). PCR detection of K-Ras mutation system contained the final concentrations of each upstream and downstream primer, blocker (Shanghai Sangon, Shanghai, China) and capture probe as 0.9, $0.9,0.9$ and $0.20 \mathrm{mmol} / 1$, respectively. The assay was set up as follows: A total of $20 \mathrm{ng}$ DNA was used and the reaction was performed in a final volume of $20 \mathrm{ml}$. Detection sensitivity and specificity of K-ras (G12V or G13D) were estimated by using serial dilutions of the corresponding mutant SW480 cell line DNA (Type Culture Collection of the Chinese Academy of Sciences, Shanghai, China) mixed with wild-type DNA (5, 1, 
Table II. Fecal K-ras mutation and serum CA19-9 in the diagnosis of pancreatic cancer.

\begin{tabular}{lccccccccc}
\hline Parameter & Sensitivity $(\%)$ & $\chi^{2}$ & P-value & Specificity $(\%)$ & $\chi^{2}$ & P-value & YI & PPV (\%) & NPV $(\%)$ \\
\hline Serum CA19-9 & 77.3 & - & - & 77.8 & - & - & 0.551 & 85.0 & 67.7 \\
Fecal K-ras & 81.8 & 0.28 & 0.597 & 81.5 & 0.06 & 0.806 & 0.633 & 87.8 & 73.3 \\
K-ras+CA19-9 & 97.7 & 16.06 & $<0.001$ & 80.9 & 0.68 & 0.513 & 0.786 & 86.1 & 94.4 \\
\hline
\end{tabular}

P-values refer to comparison with serum CA19-9. YI, Youden's index; PPI, positive predictive value; NPV, negative predictive value; CA, cancer antigen.

Table III. Association of the presence of fecal K-ras mutations with certain biomarkers for pancreatic cancer.

\begin{tabular}{|c|c|c|c|}
\hline \multirow[b]{2}{*}{ Variable } & \multicolumn{2}{|c|}{$\begin{array}{c}\text { Fecal K-ras } \\
\text { point mutations }\end{array}$} & \multirow[b]{2}{*}{ P-value } \\
\hline & Yes & No & \\
\hline Sex & & & 0.367 \\
\hline Male & $34(73.9)$ & $12(26.1)$ & \\
\hline Female & $36(81.8)$ & $8(18.2)$ & \\
\hline Age (years) & $67.00 \pm 10.63$ & $69.25 \pm 11.33$ & 0.451 \\
\hline Clinical manifestations & & & 0.152 \\
\hline Yes & $40(76.9)$ & $12(23.1)$ & \\
\hline No & $32(88.9)$ & $4(11.1)$ & \\
\hline Location & & & 0.396 \\
\hline $\begin{array}{l}\text { Pancreatic head } \\
\text { and neck }\end{array}$ & $46(79.3)$ & $12(20.7)$ & \\
\hline $\begin{array}{l}\text { Pancreatic body } \\
\text { and tail }\end{array}$ & $26(86.7)$ & $4(13.3)$ & \\
\hline Tumor diameter $(\mathrm{cm})$ & $4.08 \pm 1.22$ & $3.50 \pm 0.73$ & 0.07 \\
\hline Differentiation & & & 0.152 \\
\hline Well & $40(76.9)$ & $12(23.1)$ & \\
\hline Poor & $32(88.9)$ & $4(11.1)$ & \\
\hline CA19-9 level (U/l) & & & 0.454 \\
\hline$\geq 37$ & $54(79.4)$ & $14(20.6)$ & \\
\hline$<37$ & $18(90.0)$ & $2(10.0)$ & \\
\hline CEA level (U/l) & & & 0.517 \\
\hline$\geq 5$ & $24(85.7)$ & $4(14.3)$ & \\
\hline$<5$ & $48(80.0)$ & $12(20.0)$ & \\
\hline TNM stage & & & 0.543 \\
\hline I+IIA & $30(78.9)$ & $8(21.1)$ & \\
\hline IIB+III+IV & $42(84.0)$ & $8(16)$ & \\
\hline
\end{tabular}

Values are expressed as the mean \pm standard deviation or $n$ and percentages. CA, cancer antigen; CEA, carcinoembryonic antigen; TNM, tumor-nodes-metastasis.

0.5 and $0.1 \%$ mutant DNA solutions were prepared). The data were collected and analyzed using ABI 7500 fast System SDS software v1.4.1 (Applied Biosystems; Thermo Fisher Scientific, Inc.). PCR data were analyzed with a manual threshold of 0.1 and baseline from 5 to 15 to obtain cycle threshold $(\mathrm{Cq})$ values for FAM and VIC channels. The assay was considered valid when the $\beta$-actin $C$ T value was $\leq 20$, the specific mutant gene CT was 21-37 (w3 copies) and all No Template Controls had an undetectable CT. PCR aliquots were also analyzed by $0.8 \%$ agorose gel electrophoresis. Relative quantification was performed using the comparative threshold cycle $\left(2^{-\Delta \Delta \mathrm{Cq}}\right)$ method (21). The qRT-PCR reaction was performed at $50^{\circ} \mathrm{C}$ for $2 \mathrm{~min}$ and $95^{\circ} \mathrm{C}$ for $30 \mathrm{sec}$, followed by 40 cycles of denaturation at $95^{\circ} \mathrm{C}$ for $5 \mathrm{sec}$ and annealing at $60^{\circ} \mathrm{C}$ for $45 \mathrm{sec}$. All reactions were performed in triplicate. The probe, primers and blockers used were as follows: Kras capture probe, 5'-CTCTATTGT TGGATCATATTCGTCCACAAAATGATTCTGAATTA-3'; G12V forward primer, 5'-ACTTGTGGTAGTTGGACCT-3'; G12V reverse primer, 5'-TAACTTGAAACCCAAGGTAC-3'; blocker, CCTACGCCACCAGCT (with 4 pentabases); G13D forward primer, 5'-GTTCTAATATAGTCACATTTTCATTAT TTTTATTATAAAGC-3'; G13D reverse primer, 5'-GTCAAG GCACTCTTGCCTAGG-3'; blocker, CTTGCCTACGCCACC A (with 4 pentabases); $\beta$-actin forward, CTCCATCCTGGC CTCGCTGT $\beta$-actin reverse primer, GCTGTCACCTTCACC GTTCC.

Statistical analysis. All statistical analyses were performed using SPSS 19.0 software (International Business Machines, Corp., Armonk, NY, USA). Continuous and categorical data are shown as the mean \pm standard deviation or the percentage, which were compared by Student's t-test and the $\chi^{2}$ test, respectively. The correlation of K-ras and CA19-9 was analyzed by the $\chi^{2}$ test and the specificity, sensitivity, Youden's index (YI), positive predictive value (PPV) and negative predictive value (NPV) were also calculated. $\mathrm{P}<0.05$ was considered to indicate a statistically significant difference.

\section{Results}

$K$-ras mutation detection in fecal DNA by nanoparticle capture probe has predictive value for pancreatic cancer. DNA was successfully extracted and validated from all fecal samples. G12V and G13D mutations in the K-ras gene were detected using the magnetic $\mathrm{s}$. The $\mathrm{CT}$ value was determined and ranged from 41.71 to 62.61 in patients carrying G12V and/or G13D mutations. Of the 88 patients with pancreatic cancer, K-ras mutations were found in $72(81.8 \%)$, including G12V $(n=64)$ and G13D $(n=8)$ (Table I). The mutation rate in samples from patients with pancreatic benign diseases was $18.5 \%$ (10/54), including G12V $(n=8)$ and G13D $(n=2)$. Among the 10 cases with K-ras mutations in the benign group, 7 cases were of chronic pancreatitis, 2 
had a pancreatic mucinous cyst neoplasm and 1 had a pancreatic serous cyst. Among them, K-ras mutations were also detected in 9 cases. No mutations were detected in the healthy controls. Taken together, the K-ras mutation rate in pancreatic cancer was significantly higher than in that in pancreatic non-malignant diseases $(\mathrm{P}<0.05$; Table I).

Detection of K-ras mutations in fecal DNA has high sensitivity and specificity for pancreatic cancer. The sensitivity and specificity of K-ras mutations in fecal samples for detection of pancreatic cancer was 81.8 and $81.5 \%$, respectively (Table II). Sixty-eight pancreatic cancer patients had $>37$ U/ml CA19-9 and the sensitivity and specificity were 77.3 and $77.8 \%$, respectively, which were not significantly different from those of the K-ras mutations ( $\mathrm{P}>0.05$; Table II). Combined detection using fecal K-ras mutations and CA19-9 had a sensitivity and specificity of 97.7 and $80.9 \%$, respectively, indicating that this combination significantly increased the sensitivity of detection of pancreatic cancer to $>95 \%(\mathrm{P}<0.05)$. The specificity was not enhanced compared with that of detection by K-ras mutations or CA19-9 alone ( $\mathrm{P}>0.05$; Table II).

K-ras mutations were not significantly correlated with any of the clinical features assessed, including age, sex, clinical manifestations, tumor size, CA19-9 and CEA level, and tumor-nodes-metastasis stage of pancreatic cancer (Table III). The K-ras mutation rate was comparable in patients with I+IIA and IIB + III + IV pancreatic cancer (78.9 vs. 84.0\%; P>0.05).

\section{Discussion}

Pancreatic cancer has a low early detection and survival rate, and radical resection is the only treatment available with curative potential. Radical surgery for a pancreatic cancer sized $\leq 2 \mathrm{~cm}$ has been reported to increase the 5-year survival rate to $19-52.9 \%(22,23)$. The early diagnosis of pancreatic cancer remains challenging and it is vital that more sensitive and specific methods are developed to detect pancreatic cancer. The present study investigated the feasibility and efficacy of magnetic nanoprobes to detect K-ras mutations in fecal samples from patients with pancreatic cancer at various stages. Magnetic nanoparticles were used to extract DNA from the fecal samples and the probe was able to specifically capture the point mutation. The results indicated that this detection was sensitive, reliable, repeatable and cheap.

The novel K-ras mutation detection method used in the present study had greater sensitivity and specificity than the previous CA19-9 method. The mutation rate of the K-ras gene in pancreatic cancer was $81.8 \%$, which is higher than that reported by previous studies $(24,25)$. Fecal DNA from pancreatic cancer patients had a higher mutation rate than that of patients with pancreatic benign diseases and healthy controls $(\mathrm{P}<0.05)$. The nanoparticle capture probe is intended to detect trace DNA content, which means that smaller clinical samples are required. The present study also found that the K-ras mutation rate in fecal DNA from patients with early pancreatic cancer $(n=19)$ was comparable with that in samples from advanced pancreatic cancer patients ( $n=25 ; 78.9$ vs. 84.0\%; $P>0.05)$. These results supported that K-ras mutations participate in the initiation of pancreatic cancer, which is consistent with previous findings. For instance, Wilentz et al (26) reported that $75-100 \%$ of pancreatic cancer patients had a mutation in K-ras codon 12, suggesting that K-ras detection may be used as an early detection marker for pancreatic cancer. In addition, K-ras mutation increased the risk of pancreatic cancer in patients with chronic pancreatitis (27), while K-ras mutations were also observed in certain benign pancreatic diseases (28). In the patient cohort of the present study, a certain percentage of cases with pancreatic benign diseases had fecal K-ras mutations. Whether K-ras mutations are an independent risk factor for pancreatic cancer should be assessed in future studies.

CA19-9 $>37 \mathrm{U} / \mathrm{ml}$ is widely acknowledged as an important serum biomarker for pancreatic cancer (29), but its sensitivity and specificity are only $70-80 \%$. In the present study, the diagnostic value of K-ras mutations in fecal samples and serum CA19-9 levels were compared. The sensitivity and specificity of K-ras mutations for pancreatic cancer detection were higher than those of CA19-9 (sensitivity, 81.8 vs. $77.3 \%$; specificity, 81.5 vs. $77.8 \%$ ), but the differences were not statistically different. Of note, the use of the two diagnostic markers K-ras and CA19-9 in combination had a significantly increased sensitivity $(97.7 \%$; $\mathrm{P}<0.05)$, and therefore represents a promising early detection method for pancreatic cancer. Although fecal K-ras mutation detection is of particular translational significance, its false-negative rate, which may result from the degradation of DNA, high content of bilirubin and limited detached tumor cells in feces, should not be neglected. These issues may be prevented by modifying the fecal DNA extraction method and multi-genetic multi-locus detection.

In summary, the novel magnetic nanoprobe system used in the present study was able to detect K-ras mutations in fecal samples of patients with pancreatic cancer with higher prevalence than that in samples from patients with benign pancreatic diseases and healthy controls. Based on these results, fecal K-ras mutation detection may be recommended for screening high-risk populations, and its combination with CA19-9 may improve the early detection rate in pancreatic cancer. The method has the advantage of being non-invasive. The present study might serve as a pilot analysis of the clinical significance of fecal K-ras mutation detection in pancreatic cancer, and its diagnostic potential still requires to be comprehensively validated in larger cohorts prior to being introduced into clinical practice. In conclusion, detecting K-ras mutation in fecal matter by novel magnetic nanoprobe may be used as a potential tumor marker for diagnosing patients with pancreatic carcinoma in the future.

\section{Acknowledgements}

The present study was supported by NSFC (grant no. 81371682), awarded to Professor Lifeng Qi and the Zhejiang Provincial Science and Technology Department (grant no. 2014C33139), awarded to Dr Zhengxiang Zhong.

\section{References}

1. Sergeant G, Vankelecom H, Gremeaux L and Topal B: Role of cancer stem cells in pancreatic ductal adenocarcinoma. Nat Rev Clin Oncol 6: 580-586, 2009.

2. Li D, Xie K, Wolff R and Abbruzzese JL: Pancreatic cancer. Lancet 363: 1049-1057, 2004. 
3. Joergensen MT, Brünner N and DeMuckadell OB: Comparison of circulating MMP-9, TIMP-1 and CA19-9 in the detection of pancreatic cancer. Anticancer Res 30: 587-592, 2010.

4. Rosty C and Goggins M: Early detection of pancreatic carcinoma. Hematol Oncol Clin North Am 16: 37-52, 2002.

5. Watanabe H, Kawakami H, Yamakawa O, Satomura Y, Ohta H, Motoo Y, Okai T and Sawabu N: Clinical usefulness of tumor markers associated with pancreatic cancer. Rinsho Byori 42: 127-138, 1994 (In Japanese).

6. Huang C, Wang WM, Gong JP and Yang K: Oncogenesis and the clinical significance of K-ras in pancreatic adenocarcinoma. Asian Pac J Cancer Prev 14: 2699-2701, 2013.

7. Minamoto T: Detection and characterization of oncogene mutations in preneoplastic and early neoplastic lesions. Methods Mol Biol 1105: 381-398, 2014

8. Dabritz J, Preston R, Hänfler J and Oettle H: Follow-up study of K-ras mutations in the plasma of patients with pancreatic cancer: Correlation with clinical features and carbohydrate antigen 19-9. Pancreas 38: 534-541, 2009.

9. Duffy MJ, Sturgeon C, Lamerz R, Haglund C, Holubec VL, Klapdor R, Nicolini A, Topolcan O and Heinemann V: Tumor markers in pancreatic cancer: A European Group on Tumor Markers (EGTM) status report. Ann Oncol 21: 441-447, 2010.

10. Shin SH, Kim SC, Hong SM, Kim YH, Song KB, Park KM and Lee YJ: Genetic alterations of K-ras, p53, c-erbB-2, and DPC4 in pancreatic ductal adenocarcinoma and their correlation with patient survival. Pancreas 42: 216-222, 2013.

11. Singh N, Gupta S, Pandey RM, Chauhan SS and Saraya A: High levels of cell-free circulating nucleic acids in pancreatic cancer are associated with vascular encasement, metastasis and poo survival. Cancer Invest 33: 78-85, 2015.

12. Fuccio L, Hassan C, Laterza L, Correale L, Pagano N, Bocus P, Fabbri C, Maimone A, Cennamo V, Repici A, et al: The role of K-ras gene mutation analysis in EUS-guided FNA cytology specimens for the differential diagnosis of pancreatic solid masses: A meta-analysis of prospective studies. Gastrointest Endosc 78: 596-608, 2013.

13. Teich $\mathrm{N}$ and Mossner J: Molecular analysis of pancreatic juice: A helpful tool to differentiate benign and malignant pancreatic tumors? Dig Dis 22: 235-238, 2004

14. Däbritz J, Preston R, Hänfler J and Oettle H: Follow-up study of K-ras mutations in the plasma of patients with pancreatic cancer: Correlation with clinical features and carbohydrate antigen 19-9. Pancreas 38: 534-541, 2009.

15. Gu J, Wang D, Huang Y, Lu Y and Peng C: Diagnostic value of combining CA 19-9 and K-ras gene mutation in pancreatic carcinoma: A meta-analysis. Int J Clin Exp Med 7: 3225-3234, 2014.

16. Loktionov A, O'Neill IK, Silvester KR, Cummings JH, Middleton SJ and Miller R: Quantitation of DNA from exfoliated colonocytes isolated from human stool surface as a novel noninvasive screening test for colorectal cancer. Clin Cancer Res 4: 337-342, 1998.
17. Klaassen CH, Jeunink MA, Prinsen CF, Ruers TJ, Tan AC, Strobbe LJ and Thunnissen FB: Quantification of human DNA in feces as a diagnostic test for the presence of colorectal cancer. Clin Chem 49: 1185-1187, 2003.

18. Qi L, Wu L, Zheng S, Wang Y, Fu H and Cui D: Cell-penetrating magnetic nanoparticles for highly efficient delivery and intracellular imaging of siRNA. Biomacromolecules 13: 2723-2730, 2012.

19. Qi L and Gao X: Quantum dot-amphipol nanocomplex for intracellular delivery and real-time imaging of siRNA. ACS Nano 2 1403-1410, 2008.

20. Yuan H, Zhang L and Zhang Y: Preparation of high efficiency and low carry-over immobilized enzymatic reactor with methacrylic acid-silica hybrid monolith as matrix for on-line protein digestion. J Chromatogr A 1371: 48-57, 2014.

21. Livak KJ and Schmittgen TD: Analysis of relative gene expression data using real-time quantitative PCR and the 2(-Delta DeltaC(T)) method. Methods 25: 402-408, 2001.

22. Schnelldorfer T, Ware AL, Sarr MG, Smyrk TC, Zhang L, Qin R, Gullerud RE, Donohue JH, Nagorney DM and Farnell MB: Long-term survival after pancreatoduodenectomy for pancreatic adenocarcinoma: Is cure possible? Ann Surg 247: 456-462, 2008.

23. Benassai G, Mastrorilli M, Quarto G, Cappiello A, Giani U and Mosella G: Survival after pancreaticoduodenectomy for ductal adenocarcinoma of the head of the pancreas. Chir Ital 52: 263-270, 2000

24. Haug U, Wente MN, Seiler CM, Jesenofsky R and Brenner H: Stool testing for the early detection of pancreatic cancer: Rationale and current evidence. Expert Rev Mol Diagn 8: 753-759, 2008.

25. Lu X, Xu T, Qian J, Wen X and Wu D: Detecting K-ras and p53 gene mutation from stool and pancreatic juice for diagnosis of early pancreatic cancer. Chin Med J (Engl) 115: 1632-1636, 2002

26. Wilentz RE, Chung CH, Sturm PD, Musler A, Sohn TA, Offerhaus GJ, Yeo CJ, Hruban RH and Slebos RJ: K-ras mutations in the duodenal fluid of patients with pancreatic carcinoma. Cancer 82: 96-103, 1998.

27. Arvanitakis M, Van Laethem JL, Parma J, De Maertelaer V, Delhaye $\mathrm{M}$ and Devière $\mathrm{J}$ : Predictive factors for pancreatic cancer in patients with chronic pancreatitis in association with K-ras gene mutation. Endoscopy 36: 535-542, 2004.

28. Pugliese V, Pujic N, Saccomanno S, Gatteschi B, Pera C, Aste H, Ferrara GB and Nicolò G: Pancreatic intraductal sampling during ERCP in patients with chronic pancreatitis and pancreatic cancer: Cytologic studies and k-ras-2 codon 12 molecular analysis in 47 cases. Gastrointest Endosc 54: 595-599, 2001.

29. Ballehaninna UK and Chamberlain RS: Serum CA 19-9 as a biomarker for pancreatic cancer-A comprehensive review. Indian J Surg Oncol 2: 88-100, 2011. 